\title{
Factors que Impide a las Pymes de la Localidad de Escárcega Ilevar a Cabo un Diagnóstico o Evaluación de su Situación
}

\author{
Dra. María del Carmen Gomez Camal \\ Nicte-ha Cosgalla Arias (Licenciado en Administración) \\ Isidro Estrella Diaz (Licenciado en Administración) \\ Instituto Tecnológico Superior de Escárcega, México
}

URL:http://dx.doi.org/10.19044/esj.2019.v15n16p96

\section{Resumen}

La presente investigación es de tipo documental y de campo, se realiza una indagación en libros, revistas, informes, páginas web y medio locales para recabar información acerca de las pymes, sus características, la importancia que han tomado a lo largo de la historia en contextos nacionales, regionales y locales, de igual manera se plantea información acerca de los factores que las afectan, y la importancia que tiene que estás entidades económicas realicen evaluaciones o diagnósticos empresariales. Mediante la investigación de campo, se recaba información acerca de las pymes de la localidad de Escárcega, como le afectan los diversos factores macroambientales y microambientales, esto con la finalidad de lograr obtener el objetivo general del estudio, el cual es determinar el factor que impide a las pymes de la localidad, realizar o no una evaluación o diagnóstico empresarial. Esta información se obtendrá mediante una encuesta que aborda temas generales de cada empresa, como le afectan los factores externos e internos y la importancia de la realización que se tiene para el crecimiento de las organizaciones. Esta herramienta tendrá un proceso de validación mediante el coeficiente del Alfa de Cronbach, que mostrará la confiabilidad que tendrá la encuesta al momento de ser aplicada. Dentro de los resultados esperados obtendremos cuantas pymes realizan diagnósticos o evaluaciones, como las realizan, las o el área que consideran que es más importante tener vigilada, el factor que las afecta al momento de tomar la decisión de usar estas herramientas y quien es el encargado de realizarlas.

Palabras claves: Factores, Pymes, Sectores, Diagnóstico empresarial, Situación de las empresas 


\title{
Factors that Prevents SMEs in the Locality of Escárcega from Carrying Out a Diagnosis or Evaluation of their Situation
}

\author{
Dr. María del Carmen Gomez Camal \\ Nicte-ha Cosgalla Arias (Bachelor of Administration) \\ Isidro Estrella Diaz (Bachelor of Administration)
}

Higher Technological Institute of Escárcega, México

\begin{abstract}
The present paper is a field and documentary investigation acquired from books, magazines, reports, web pages, and local media for the purpose of obtaining information about SMEs. It also contains a compilation of its characteristics, the importance of SMEs based on the history in national, regional, and local contexts, the equal way this brings information in regard to the factors that affect them, and how important it is for economic entities to realize the evaluations or managerial diagnosis. Through field research, information was obtained about the SMEs of the locality of Escárcega. It also reveals how they are affected by the diverse macro-environmental and microenvironmental factors. Therefore, this paper focuses on determining the various causes that prevent the SMEs of the locality to realize an evaluation or managerial diagnosis. This information was obtained through an inquest that approaches general topics of every company, how the external and internal factors affect them, and the importance of the accomplishment they have towards the growth of the organizations. This tool was validated using Cronbach's Alpha coefficient. It shows the reliability that the inquest will have at the moment of being applied. The results obtained show the number of SMEs realized, diagnosed or evaluated, the area that they consider the most important to be monitored, the factor that concerns them at the moment of making the decision in using these tools, and who is the manager in charge of realizing them.
\end{abstract}

Keywords: Factors, SME's, Sectors, Business diagnosis, Situation of Companies 


\section{Introducción}

Las pymes han tomado gran importancia a medida que las economías van cambiando, siendo este uno de los temas con mayor debate en la actualidad, y además de contar con deficiente información acerca de la situación actual de las empresas, se ha decidido realizar una investigación en donde se pueda determinar, cómo las pymes de la localidad de Escárcega, realizan diagnósticos o evaluaciones de su situación y en caso de no realizarlas, cuál es el factor influyente para tomar esta decisión.

A lo largo de este estudio se analizarán temas como los factores que afectan a las pequeñas y medianas empresas en Escárcega, su importancia, características y como ha sido su evolución a través del tiempo, de igual manera se estudiaran las características de los diagnósticos y las evaluaciones que las pymes deben llevar a cabo para determinar correctamente su situación, todo esto se realizará mediante una metodología sustentada que incluye el análisis de las opiniones de diferentes autores, buscando obtener información clara y precisa que apoye el desarrollo de una herramienta confiable de obtención de datos, los cuales posteriormente serán analizados y discutidos, esperando obtener el factor que determina la realización de un diagnóstico o evaluación empresarial en las pequeñas y medianas entidades económicas de la ciudad de Escárcega.

\section{Materiales y Métodos}

De acuerdo al proceso de investigación, se desarrollan actividades que apoyan la búsqueda de los resultados a los objetivos planteados, tanto generales como específicos.

La metodología a seguir para dar respuestas a las preguntas de investigación, se realiza mediante dos etapas.

La primera es una investigación documental, esta se llevó a cabo mediante la consulta de libros, tesis, artículos, paginas especializadas en internet y revistas que proporcionaran información valiosa acerca de factores internos y externos, evaluaciones y diagnósticos para las pymes.

La segunda parte del proyecto se conforma por una investigación de campo a través de la estructuración de una herramienta de recolección de datos, de manera que aborde los temas de interés para el estudio y en base a ello se realizaron una serie de interrogantes.

El cuestionario se constituyó de forma que nos arrojara información desde los aspectos más generales hasta los más particulares, y fue conformado por treinta y seis preguntas, teniendo cuatro respuestas definidas y ponderadas de acuerdo a la escala de Likert y sustentada por Rendís Likert en 1932; que trata de un enfoque vigente y bastante popularizado que consiste en un conjunto de ítems presentados en forma de afirmaciones o juicios, ante los cuales se pide la reacción de los participantes. Es decir, se presenta cada afirmación y se 
solicita al sujeto que externe su reacción eligiendo uno de los puntos o categorías de la escala (Sampieri, 2014).

Como ya se mencionó anteriormente, el cuestionario fue estructurado exclusivamente para esta investigación, por lo tanto, esta herramienta debió de pasar por un proceso de validación que diera la certeza de que se utilizó un instrumento el cual proyectara información viable y confiable.

Para obtener el grado de confiabilidad se eligieron veinte pymes de las cincuenta disponibles, de los tres sectores (industria, comercio y servicios), los resultados servirán para ejecutar la matriz de validación por alfa de Cronbach.

Este método de consistencia interna permite calcular y visualizar por medio de un coeficiente la fiabilidad del instrumento a usar en nuestra recabación de datos a través de un conjunto de ítems previamente elaborados, en el cual, con el alfa de Cronbach se asumen que los ítems miden el mismo constructo y están correlacionados de manera idónea.

De esta manera el coeficiente dado por el alfa debe encontrarse lo más cercano al valor de 1, ya que de ser así se puede decir que la consistencia es mayor.

Para evaluar los criterios de alfa de Cronbach, hay que tener en cuenta los siguientes criterios (Darren George, 1995).

1. Coeficiente alfa $>.9$ es excelente

2. Coeficiente alfa $>.8$ es bueno

3. Coeficiente alfa $>.7$ es aceptable

4. Coeficiente alfa $>.6$ es cuestionable

5. Coeficiente alfa $>.5$ es pobre

6. Coeficiente alfa $<.5$ es inaceptable

Podemos encontrar que la fórmula estadística del alfa de Cronbach es la siguiente (Leal, 2014):

$$
\alpha=\frac{\mathrm{k}}{\mathrm{k}-1}\left[1-\frac{\Sigma s i^{2}}{\mathrm{St}^{2}}\right]
$$

Donde:

$\alpha=$ Alfa de Cronbach

$\mathrm{K}=$ Número de ítems

$\Sigma \mathrm{Si}^{2}=$ Sumatoria de las varianzas de cada ítem

$\mathrm{St}^{2}=$ Varianza del total 
Sustituyendo los datos tenemos que:

$\mathrm{K}=36$

$\Sigma \mathrm{S}^{2} \mathrm{i}=36.661$

$\mathrm{St}^{2}=1040.742$

Sustituyendo en la formula tenemos que:

$\alpha=\frac{36}{36-1}\left(1-\frac{36.661}{1040.742}\right)$
$\alpha=\frac{36}{35}[1-0.035]$

$\alpha=1.0286[0.9648]$

$\alpha=1.0286 * 0.9648$

$\alpha=0.99$

Por lo tanto, tenemos que el Coeficiente alfa $=0.9$, se determina que es excelente.

A continuación, se presenta la herramienta de recolección de datos utilizado para la investigación.

\section{5}

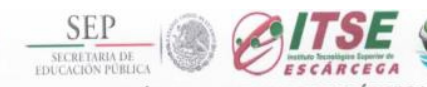

INSTITUTO TECNOLÓGICO SUPERIOR DE ESCÁRCEGA.

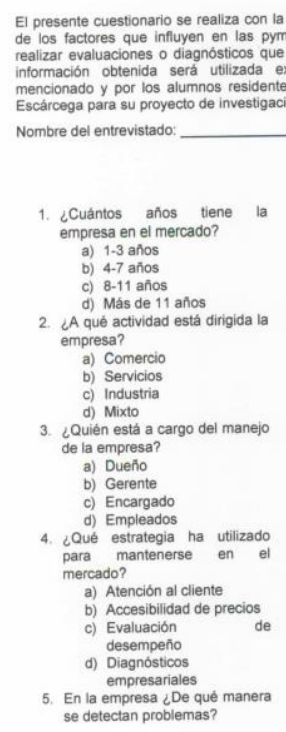

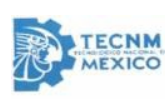

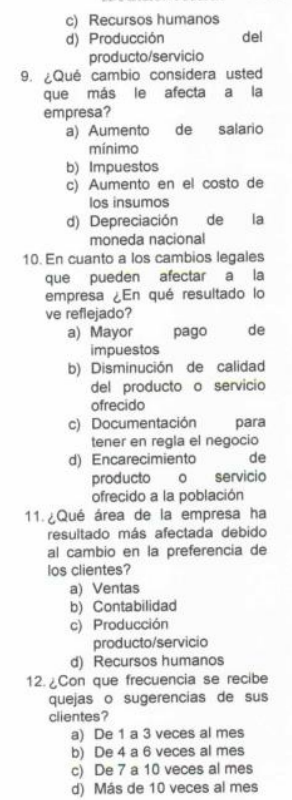

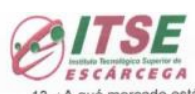

13. ¿A qué mercado está dirigido su producto?

a) Público en general

Sexo en especifico C) Mayores de edad

4. ¿Qué método utilizo para

14. ¿Qué método utilizo para
conocer las caracteristicas de

su mercado?

a) Análisis de mercado

besoria externa

c) Análisis de factibilidad d) Decisión empirica

15. ¿Qué tan accesible considera

que es su producto y/o servicio?

a) Muy accesible

b) Accesible

c) Poco accesible

16. Considera usted que,
Muy

16. Considera usted que,
comparación de años pasados,

el consumo de su

producto/servicio ha:

a) Aumentado mantenido

iguales

c) Disminuyeron

d) No tiene conocimiento

7. ¿Cuál considera usted que es

uno de los principales

problemas, por el que los

clientes han disminuido su

úttimos ańos?

a) Escaso aumento de

b) Depreciación de la

moneda

c) Inflación

d) Aumento de impuestos a

los productos o insumos

18. ¿Cómo determina el precio de

su producto o servicio? 

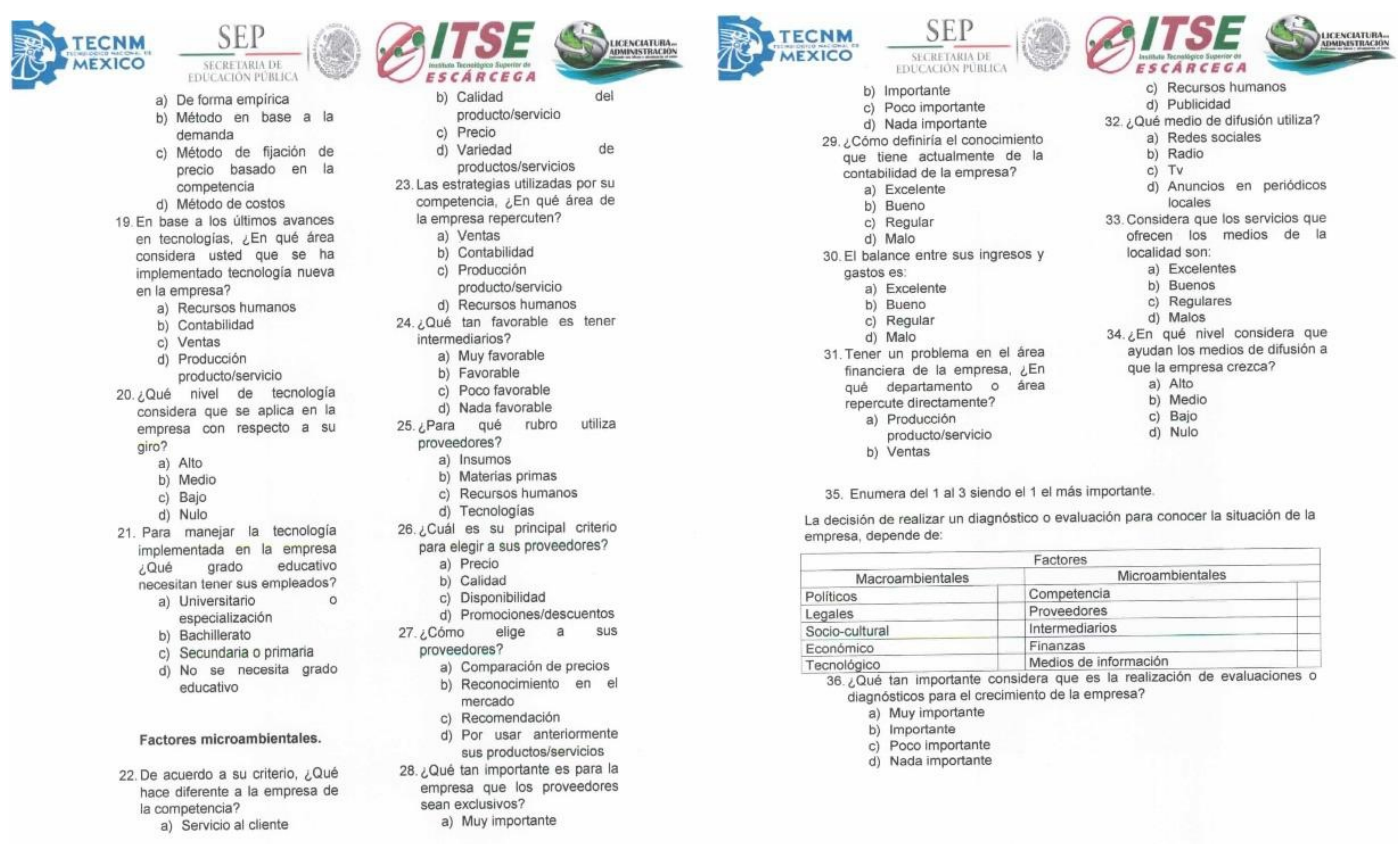

\section{Resultados}

De acuerdo con (Juan Carlos Alcaide, 2013), la descripción de la situación actual de una pyme, constituye el punto de partida del plan de marketing, logrando recopilar la máxima información de los factores que afectan a las pymes. Para (Laura Fischer, 2011) el sistema de la mercadotecnia de una empresa debe operar dentro de una estructura de fuerzas que pueden ser externas o internas a la empresa. De igual manera, (Norma Hernández, 2018) nos dice que un departamento de mercadotecnia funciona en un entorno que se ve afectado por factores externos a la organización, estos factores pueden ser fuerzas "macroambientales" o "microambientales".

Los resultados que se obtuvieron mediante la recabación de datos, demuestran la situación actual de las pymes en la localidad de Escárcega, en estos, podemos observar las deficiencias y virtudes de las empresas, que sirven como la base para emitir un juicio de la situación que atraviesan, en este apartado podemos encontrar todos los datos de forma detallada y precisa, arrojados a través de la herramienta que se empleó para el estudio de las pymes.

Este proceso de investigación, se basa en una muestra de 50 pymes de la localidad de Escárcega y se obtienen los siguientes resultados.

El 72\% de las Pymes, que representa a 36 empresas de la localidad, cuentan con más de 11 años en el mercado, la mayoría ha sabido afrontar los problemas, pero no ha podido ir más allá, es decir, crecer y posicionarse como 
una empresa reconocida dentro de la ciudad. Mientras tanto, un 36\% de las pymes encuestadas se dedican a la prestación de servicios, el $34 \%$ al comercio, el $10 \%$ a la industria y un $20 \%$ tiene un giro mixto, por lo que hacen del comercio y los servicios, las actividades económicas principales de Escárcega, además, 32 negocios son dirigidos y manejados por sus propietarios, esto se debe a que estas empresas son de tipo familiar.

En consecuencia, un $36 \%$ de las pequeñas y medianas organizaciones, tienen como estrategia para mantenerse en el mercado, la atención al cliente, dejando solo a una que utiliza diagnósticos empresariales, el resto utiliza la accesibilidad de precios; esto se debe a que un $64 \%$ detecta problemas gracias a las quejas y sugerencias de sus consumidores, el 18\% lleva a cabo el análisis de instrumentos propios, mientras las nueve empresas restantes ejecutan evaluaciones o diagnósticos; Esto conlleva a que el 46\% de los empresarios elaboren controles internos que les brinden información y de esta forma, solventar la falta de conocimiento para identificar situaciones de riesgo, el $18 \%$ practica las auditorías internas, el $4 \%$ busca apoyo en profesionales externos a la empresa y el resto no utiliza ningún método para tener conocimiento de su situación; no obstante, el $40 \%$ considera que es importante realizar evaluaciones o diagnósticos en la producción del producto o servicio, a pesar de que 33 empresas se enfocan en el servicio al cliente, tan solo 7 utilizaría estas herramientas en el área de recursos humanos.

Por lo que se refiere a la perspectiva que tiene el empresario escarceguense, acerca del ambiente político que enfrenta México en la actualidad, estos afirman que al 76\% le afecta en el área de ventas, 12\% en contabilidad, 10\% producción y/o servicio, el porcentaje restante, en el área de recursos humanos, esto sumado al aumento en el costo de los insumos que afecta al 54\% de los negocios, además, el aumento de los impuestos a las empresas perjudica al $38 \%$ de la población, por lo tanto, provoca el encarecimiento del producto y/o servicio culminando en el declive de las ventas. Para el $82 \%$ de los empresarios, esta área es la principal afectada en cuanto al cambio en la preferencia de los clientes, mismos que realizan quejas y sugerencias de mejoran en promedio de 1 a 3 veces al mes para 41 empresas, el sobrante recibe críticas entre 4 y 10 veces al mes.

Del total de pymes, 24 realizaron su puesta en marcha de forma empírica, es decir, sin elaborar ningún tipo de estudio para conocer su mercado, aun así, han sabido enfocarse a un segmento de clientes específico siendo el $92 \%$ de las empresas las que brindan su producto y/o servicio al público en general, $\mathrm{y}$ estas ofrecen a sus clientes productos accesibles, en un $70 \%$ y muy accesibles con un $30 \%$, debido a que tratan de mantener un precio justo para sus clientes. Por consiguiente, 18 pymes consideran que sus ventas han disminuido en comparación de años pasados, y 25 empresas opinan que uno de los principales factores influyentes en el poder adquisitivo del cliente, es el aumento del 
salario mínimo. Para solventar este problema, el 58\% de las organizaciones establecen precios por medio del método de costos, esto con el fin de establecer un rango justo de ganancias que no dañe la economía de sus consumidores y por supuesto, lograr tener liquidez dentro de la misma.

En el ámbito tecnológico, el $40 \%$ de las empresas de la localidad han implementado tecnología nueva en el área de producción, el $72 \%$ considera que aquella tecnología implementada es de nivel medio y, por lo tanto, el 32\% contrata personal con estudios básicos de secundaria o primaria para realizar los nuevos procedimientos, seguido muy de cerca de un $30 \%$ que contrata empleados con nivel medio superior, para manejar la maquinaria implementada, puesto que toman cursos o capacitación dentro de las empresas.

De acuerdo al criterio de los empresarios, el $46 \%$ de las organizaciones son diferentes de su competencia gracias a la calidad del producto y/o servicio que ofrecen a la población, esto a pesar que las estrategias que la misma competencia utiliza, repercute en el área de ventas de un $86 \%$ de las pymes encuestadas.

Para los dueños, gerentes, encargados y empleados el tema de los intermediarios es un área que desconocen y no tiene suficiente importancia para ellos, en su mayoría, las empresas no cuentan con intermediarios, no obstante, el $32 \%$ de las pymes encuestadas consideran que sería favorable para sus ventas, el $24 \%$ dice que les resulta poco favorable, mientras que, para 8 empresas, este elemento sería de gran utilidad, las pymes restantes dicen que tener intermediarios no es beneficioso.

Otro factor importante para que las empresas continúen trabajando son los proveedores, el $64 \%$ de las pymes los utilizan para abastecer sus insumos y el $36 \%$ restante para sus materias primas, como consecuencia, el $58 \%$ utiliza como principal criterio para la elección de sus proveedores la calidad de los productos o servicios que les ofrecen, el $48 \%$ restante se divide entre calidad, disponibilidad y promociones o descuentos, esto sin dejar a un lado que el $44 \%$ elige a sus abastecedores mediante comparación de precios, el $36 \%$ por haber usado anteriormente los productos o servicios que ofrecen y el $20 \%$ se inclina por el reconocimiento en el mercado. También es importante mencionar que para un 36\% de la población pyme es muy importante que sus proveedores sean exclusivos, puesto que, compartir este elemento dejaría al mismo nivel a sus respectivas competencias.

Siguiendo con los resultados del estudio, el 70\% de los empresarios dicen tener un buen conocimiento de su contabilidad, el 20\% regular y el $10 \%$ tiene un conocimiento excelente de sus finanzas, de igual manera, el balance entre ingresos y gastos es bueno para el $74 \%$ de las empresas encuestadas, pero cuando las organizaciones presentan un problema en su economía, la principal área afectada son las ventas en un $38 \%$, porque si no cuentan con la liquidez suficiente, no pueden adquirir los insumos necesarios para brindar el servicio 
que los clientes deseen, seguido del área de producción con 32\%, recursos humanos en $28 \%$ y al $2 \%$ restante le afecta en la publicidad.

Por otro lado, un $86 \%$ de las empresas que formaron parte del estudio utilizan las redes sociales como medio para darse a conocer en la localidad, un $12 \%$ los anuncios en periódicos locales y el $2 \%$ utiliza publicidad por televisión, esto se debe a que solo 58\% afirma que los servicios que estos medios ofrecen, son buenos, debido a que no llegan a captar la atención del público que los empresarios esperan, de igual manera, el 44\% de los dueños, afirman que los servicios de publicidad ayudan a crecer a las empresas en un nivel medio. "Solo las anuncian cuando pasan cerca de la empresa, así la gente que queremos que conozca el negocio nunca lo hace”. Lucia Trejo, encargada de la papelería "El triunfo".

Para finalizar, tenemos uno de los resultados más importantes de la investigación y es precisamente, determinar el factor que las pymes toman en cuenta para decidir si realizan una evaluación o diagnostico que les ayude a conocer la situación en la que se encuentra la empresa, y tenemos que un $38 \%$ piensa primero en sus finanzas, es decir, si cuentan con el recurso económico necesario para contratar a expertos, esto se debe que como ya se expuso anteriormente en la mayoría de las pymes solo se detectan problemas por medio de las quejas y sugerencias de los clientes y no aplican métodos más confiables como una evaluación o diagnostico empresarial; o aplicar las estrategias que resulten necesarias para contrarrestar los problemas que surjan, puesto que muy pocos empresarios están dispuestos a invertir en un plan que probablemente funcione.

Seguidamente se determinó que el segundo factor más importante con un $20 \%$ de aceptación, es el económico, el medio en el que las pequeñas y medianas empresas se desenvuelven en la ciudad es una limitante, debido a que, si los consumidores no tienen la suficiente liquidez para comprar sus productos o servicios, ellos tampoco tienen para abastecerse, seguir en funcionamiento $\mathrm{y}$, por lo tanto, no tendrán para contratar a expertos que puedan evaluar y diagnosticar problemas. "Si la economía baja, nadie consume, nadie vende" Hilario García, propietario de pizzería "La troje".

El tercer factor que impide a las pymes locales sobresalir, es el factor tecnológico con un 16\% de aprobación, muy pocos propietarios o encargados tienen un grado educativo que les permita manejar la tecnología actual, herramientas que pueden llegar a ser muy fáciles de utilizar y ayudarían a la detección de problemas de manera más confiable, esto aunado a que no cuentan con la economía suficiente para emplear equipos sofisticados, e incluso, el utilizar una computadora para tener registros básicos, se ha convertido en un avance tecnológico caro para las pymes.

Concluimos este apartado con una de las afirmaciones más importante y que a su vez nos muestra contrariedad de la realizad, y es que un $62 \%$ de los 
dueños y encargados encuestados creen que la realización de evaluaciones o diagnósticos es importante para que las empresas crezcan, y el restante 38\% dice que es muy importante, sin embargo, solo un $10 \%$ realiza evaluaciones y un $8 \%$ algún tipo de diagnóstico y esto por medio de controles internos que los mismos dueños elaboran.

A continuación se presentan las graficas obtenidad durante el proceso de analisis de datos.
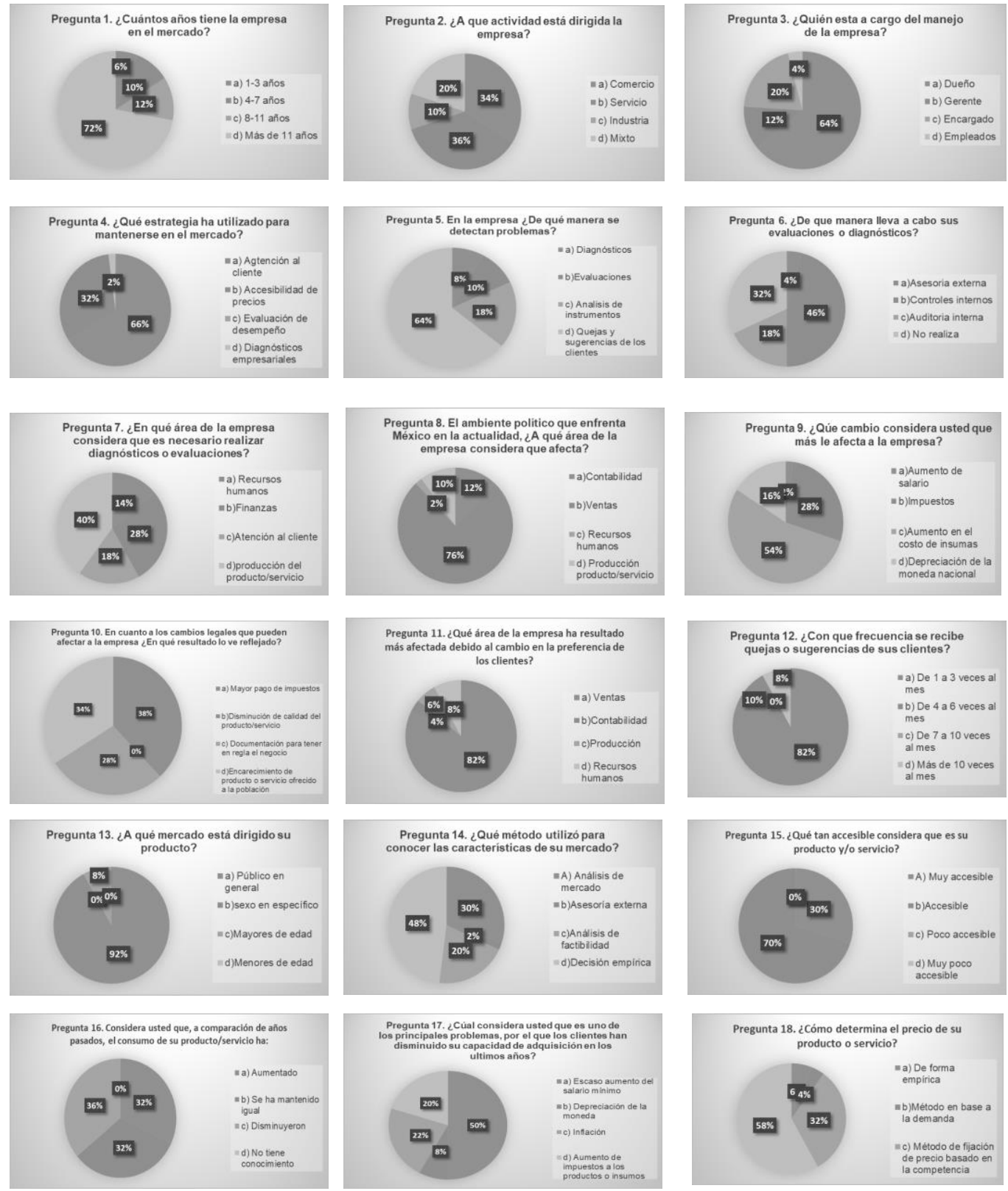

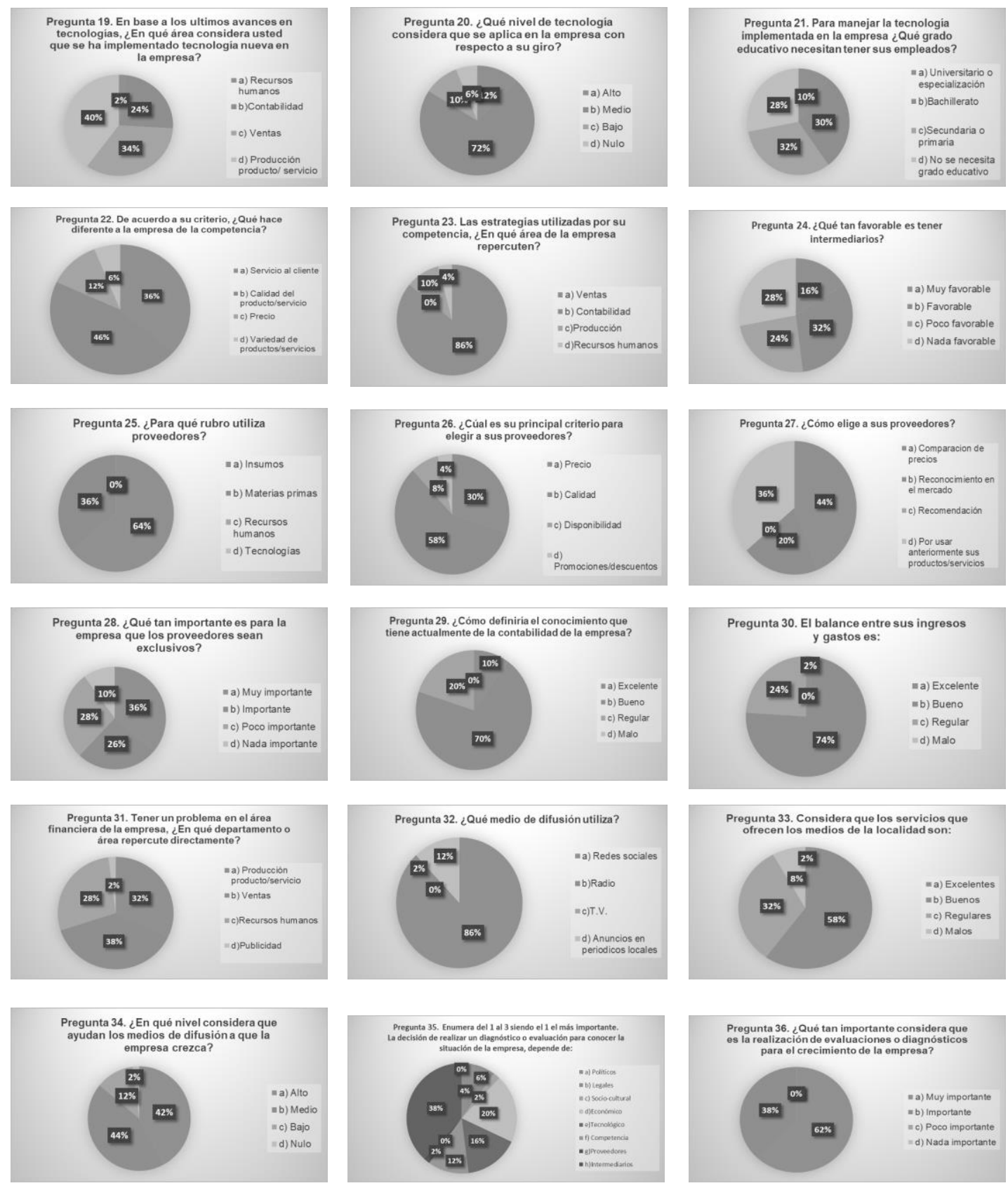

\section{Conclusión}

De acuerdo al proyecto realizado en la localidad de Escárcega, se procedió a analizar una muestra de 50 empresas de las 95 pymes existentes. 
Este proceso de investigación tuvo por objetivo, conocer, analizar y evaluar el número de empresas que llevan sus evaluaciones y diagnósticos, mediante instrumentos que determinan el buen funcionamiento de las pequeñas y medianas empresas, teniendo como conclusión que de nuestra muestra solo una lleva a cabo este proceso y 49 no las realizan, esto debido a que existen diversos factores que juegan un papel importante en el crecimiento y desarrollo de este tipo de entidades económicas.

Para este estudio se analizaron los factores macroambientales y microambientales llegando a la conclusión de que el elemento más importante a considerar por las pymes, es el financiero, esto como consecuencia de que los empresarios, piensan que invertir en un proceso de evaluación o diagnóstico pondrá en riesgo su patrimonio, y el pedir financiamiento se ha convertido en un tema tabú, haciendo que el temor de no lograr obtener los ingresos suficientes para regresar el préstamo, les hace creer, que no pueden estar en una situación mejor a la que se encuentran actualmente.

El segundo factor que ocasiona la falta de aplicación de estos métodos, es la economía del medio en el que se desarrollan las empresas, debido a que, si los consumidores no cuentan con la capacidad de adquisición necesaria, no pueden comprar los productos y servicios que estas ofrecen.

Por ultimo tenemos al aspecto tecnológico como tercer factor influyente al momento de tomar la decisión de evaluar o diagnosticar la situación empresarial, y es que, este elemento no se ha hecho presente en la mayoría de las pymes locales, a causa de que los dueños y encargados, no tienen el conocimiento necesario para su implementación, esto sumado a la carencia actual en sus finanzas.

Esta investigación responde a las preguntas de investigación.

1.- ¿Realizan las pymes de la localidad de Escárcega algún tipo de evaluación o diagnóstico para determinar su situación? No, solo una empresa de las 50 encuestadas utiliza los diagnósticos empresariales como herramienta para la detección de situaciones de mejora.

2.- ¿Qué factor determina realizar o no, una evaluación o diagnostico a las pymes locales? El factor financiero, seguido del factor económico y el tecnológico.

\section{References:}

1. Adriana Meza, P. C. (2009). Mi espacio. Recuperado el septiembre de 2018, de Desde la investigación: https://www.infosol.com.mx/espacio/Articulos/Desde_la_Investigaci on/El-Diagnostico-Organizacional-elementos.html\#.W7A7SugzbIU

2. Chiavenato, I. (2006). "Introducción a la Teoría General de Administración" (séptima ed.). Mc Graw-Hill. 
3. ENAPROCE (2016). BOLETÍN DE PRENSA NÚM. 285/16. Aguascalientes: INEGI.

4. Equipo de redacción profesional. (2015). Tipos de cosas. Recuperado el 09 de 2018, de https://www.tiposdecosas.com/evaluacion.html

5. Escobar, R. (2012). 5 días. Pasión por los negocios. Obtenido de http://www.5dias.com.py/la-importancia-del-diagnstico-empresarial/

6. Faccio, G. (2017). EUAX. Recuperado el 09 de 2018, de https://www.euax.com.br/2017/09/3-etapas-essenciais-dodiagnostico-de-performance-organizacional/

7. García, J.C. (2010). https://pymesdemexico.wordpress.com. Obtenido de https://pymesdemexico.wordpress.com/2010/11/10/historia-de-laspymes/

8. Glenda Armas Noda, S. M. (2009). Propuesta de una herramienta diagnóstico para evaluar la comprensión de la Gestión de la. Ciencias de la información, 40(3), 22. Obtenido de http://www.redalyc.org/pdf/1814/181421561002.pdf

9. Ayuntamiento de Escárcega (2015). Plan de desarrollo municipal de Escárcega 2015-2018. Escárcega.

10. IASB (2009). NIIF para PYMES. Londres: IASCF Publications Department.

11. INEGI (2014). Micro, pequeña, mediana y gran empresa: estratificación de los establecimientos. Aguascalientes: INEGI.

12. Jesús, J.L. (2018). Facebook. Obtenido de Escárcega Real: https://www.facebook.com/permalink.php?story_fbid=20257230008 $05115 \& \mathrm{id}=1163391420371615 \&$ \&n_ $=\mathrm{K}-\mathrm{R}$

13. Juan Carlos Alcaide, S. B. A. (2013). MARKETING Y PYMES: Las principales claves de marketing en la pequeña y mediana empresa. (Primera ed.). Madrid.

14. Laura Fischer, J. E. (2011). Mercadotecnia (Cuarta ed.). México: McGraw-Hill.

15. Leal, J. P. (2014). https://asesoriatesis 1960.blogspot.com. Obtenido de https://asesoriatesis1960.blogspot.com/2014/07/coeficiente-alfade-cronbach.html

16. Mendivil, A. B. (2015). Análisis de los factores internos y externos que impactan a pequeñas y medianas empresas constructoras de edificación de vivienda en Culiacán Sinaloa, énfasis en factor financiero. México: Universidad Nacional Autónoma de México.

17. Molina, M. T. (2015). Gestiopolis.com. Obtenido de https://www.gestiopolis.com/definicion-y-objetivos-de-la-evaluaciondel-desempeno/

18. Norma Hernández, J. http://www.microempresa.asenz360.com.

A. (2018).

Obtenido de 
http://www.microempresa.asenz360.com/download/Las\%20PyMEs\% 20Mexicanas.pdf

19. Pelayo, V. G. (2013). Nexian. Obtenido de https://nexian.es/importancia-de-la-evaluacion-en-la-formacion-deempresa/

20. Periódico Excélsior (2010). Colegio de contadores públicos de México A.C. https://www.ccpm.org.mx/avisos/22112010empresa.pdf

21. Sampieri, R. H. (2014). Metodología de la investigación. México: McGrawhill.

22. Ucha, F. (2009). Definición ABC. Obtenido de https://www.definicionabc.com/general/evaluacion.php

23. Yesi, U. (2014). Wordpress.com. Recuperado el 09 de 2018, de https://jessicony.wordpress.com/2014/11/27/importancia-deldiagnostico-empresarial/ 